\title{
A Robust Method for Vehicle License Plate Recognition based on Harries Corner Algorithm and Artificial Neural Network
}

\author{
Sangita Kumari \\ Student \\ Dr. B. R. Ambedkar \\ NIT Jalandhar
}

\author{
D. K. Gupta \\ Associate Professor \\ Dr. B.R. Ambedkar \\ NIT Jalandhar
}

\author{
Raj Mohan Singh \\ Assistant Professor \\ Dr. B. R. Ambedkar \\ NIT Jalandhar
}

\begin{abstract}
Automatic vehicle license plate recognition system plays an important role in Intelligent Transportation System. Even though many license plate recognition methods have been proposed in the past, further scope for improvement still exists. This paper has proposed a new approach to license plate recognition system based Harris corner detection algorithm. In this paper connected component analysis is used for character segmentation and artificial neural networks for character recognition. It is observed from the experiments that the developed system successfully identifies and recognizes the vehicle number plate under different illumination conditions and independent of orientation and scale of the plate.
\end{abstract}

\section{General Terms}

Digital Image Processing, Pattern Recognition, Computer Vision.

\section{Keywords}

Intelligent Transportation System, Number Plate, Connected Component Analysis, Sliding Window, Corner Point..

\section{INTRODUCTION}

Intelligent Transportation System has been an important research area that impact people's quality of life and improve road safety and mobility. It can reduce the accident by communicating information to driver about dangerous conditions. The automatic license plate has an important role in the intelligent transport system. It is used for vehicle management, security, congestion control, access control, unattended parking, traffic law enforcement and in the vehicle behavior monitoring system. The first automatic license plate recognition was established in 1976 at the Police Scientific Development Branch in the UK [1]. Typical automatic license plate recognition System consists of four steps: image acquisition, license plate detection, character segmentation, and character recognition.

This paper demonstrates a new approach that can manage various light condition (such as brightness, nightshade, shadow) and also with distortion condition. For this reason captured images of different types of vehicles in different illumination condition have been dealt to signify the validity of the proposed method.

\section{LITERATURE SURVEY}

Generally, license plate recognition system works in three prinicipal steps, plate detection, character segmentation, and character recognition. The structure of number plate varies from country to country. Hence, many of the algorithms for plate identification and recognition are dependent on the structure of the plate. Therefore, some of the research defines a fixed structure of the number plate and propose algorithms to recognize a certain type of number plate. This paper proposes a new approach for license plate recognition and gives a brief overview of the existing systems.

Kaushik Deb et. al. [2] proposed a method for vehicle license plate recognition based on sliding concentric windows and artificial neural network. This paper includes methods like gray scale conversion, binarization, detecting candidate region, filtering color, tilt correction, segmentation, and recognition. To detect the candidate region authors used the sliding concentric windows. Further, they discussed about authenticating the candidate region color using HIS color model. They used vertical projection for character segmentation and artificial neural network for character recognition.

Kumar Parasuraman et. al. [3] proposed a method for Indian vehicle license plate extraction and character segmentation. This paper includes methods such as gray scale conversion, edge detection, dilation, vertical projection, thresholding, filtering, and smoothing of the image. To detect the plate region, the authors applied the connected component analysis and then, they apply dilation to extract the true plate region. The authors filtered the plate region to remove the unwanted region of the plate.

Shouyuan $\mathrm{Yu}$ et. al. [4] proposed a method for license plate localization based on wavelet transform and EMD analysis. The proposed scheme consists of several steps like preprocessing (It includes Histogram equalization and Gaussian filtering), extract the detail of the image and EMD analysis. In the proposed system, the authors applied the two dimensional wavelet transformation to an image, which can separate the detail information of the license plate from the whole vehicle images without losing the information about the license plate. EMD analysis is used to extract the true license plate region.

Edson Cavalcanti Neto et. al. [5] proposed a system for vehicle identification using a new embedded plate recognition system. The proposed system is implemented in c language on Linux operating system, without graphical interface. The first step of the proposed system is image filtering using a canny edge detection operator, which is used to enhance the edges of the object in the image. After that, they apply the Hough transform to find all possible rectangles in the image. Aspect ratio is used to find the true plate region. Next step is the character segmentation and identification. For character 
segmentation, thresholding and filtering is applied to the image. Neural Network is used for character recognition.

Y.Y Nguwi et. al. [6] proposed a method for number plate recognition in noisy images. The system is composed of detection sub module and recognition sub module. In detection module the image is filtered and then the system identifies the plate region. The first step of detection module is preprocessing. In preprocessing the image first converted into a gray scale image so as to eliminate the hue and saturation formation while retaining the luminance. To further enhance the image the median filter is used. After that, morphological operation is applied to detect the number plate region. Erosion operation is used to shrink the object and opening consists of erosion and dilation. For recognition sub module neural network is used.

Shuang Qiao et al. [7] discussed about the process of license plate character segmentation. This is achieved by performing image binarization followed by character segmentation using the improved Bernsen method for binarization. In this work first convert the image in binary image using improved Bernsen method. After that eliminated the border of the plate, for which the authors used the line shape filter. The line shape filter reduces the dependency on tilt correction. For character segmentation the authors used vertical and horizontal projection

Pratiksha Jain et al. [8] discussed the process of automated license plate recognition, which contains some image preprocessing steps followed by OCR recognition. It used an edge detector to obtain the characters from the number plate, but it is more time consuming for vehicle plate processing.

Reza Azad et. al. [9] proposed a method for license plate Recognition using the edge detection. In this paper, the authors applied the Sobel operator to detect the vertical edges and then applied the morphological operation to extract the plate. After that, they applied tilt correction on the plate region. After tilt correction, they applied the thnning operation. For feature extraction the authors divided the image into subimages.

Abbas M. Al-Ghaili et. al. [10] proposed a method for license plate detection based on vertical edge detection. In this paper the authors proposed a fast vertical edge detection algorithm based on the contrast between the grayscale values. In this paper, first, the colored Input image is converted into a gray scale image, and then adaptive thresholding is applied for binarization. After that, the ULEA (Unwanted line elimination algorithm) is applied to remove the noise and to enhance the image. Next, the vertical edge detection algorithm is applied to extract the vertical edges. After that, statistical analysis is used to find the true license plate region.

\section{PROPOSED WORK}

Our proposed system architecture is shown in Figure 1, which correctly recognizes the number plate from a vehicle image which is captured throughout the several timings and with different illumination conditions. The proposed system is independent of the orientation of the plate. Our proposed system consists of several processes: gray scale conversion, image binarization, image enhancement, plate localization, character segmentation and character recognition.

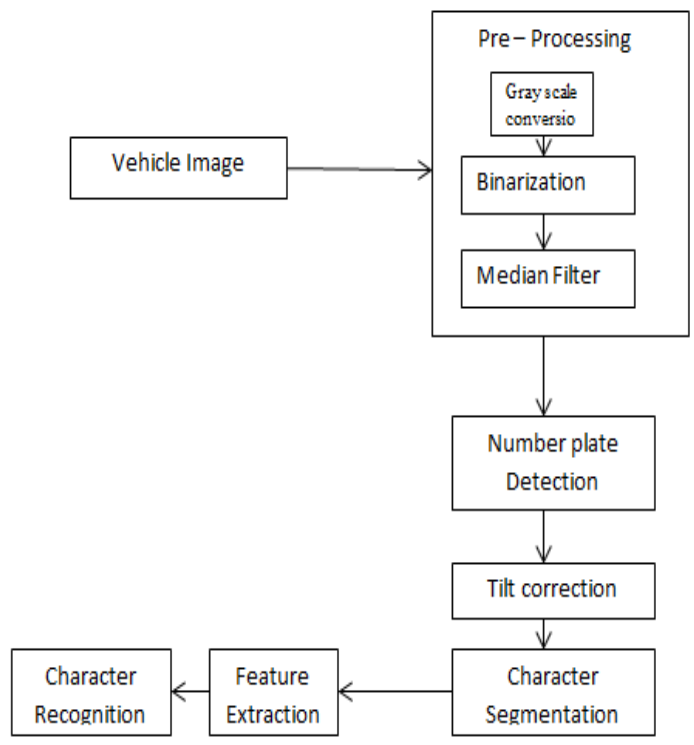

Fig 1: System Architecture.

\subsection{Preprocessing}

The first step of our system is preprocessing. The preprocessing consists of several processes: gray scale conversion, binarization, and image enhancement. Initially the RGB image of the vehicle is captured by the camera and then convert the RGB image in gray scale image because of this the speed of the system is increased.

After the grayscale conversion in this paper adaptive thresholding is applied to convert the gray scale image into a binary image. After the binarization the median filter is applied to remove the noise from the vehicle image. Median filter is a non- linear filter and it replaces the gray value of a pixel by the median of the gray value of the pixel.

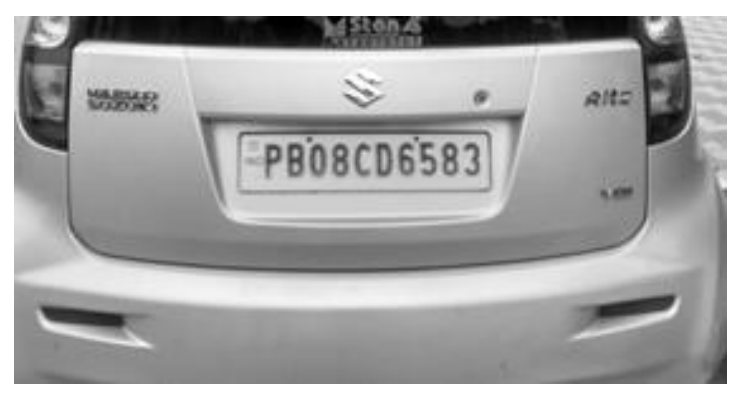

Fig 2: Gray scale image.

\subsection{Plate Detection}

After the preprocessing step, the Harries Corner detection algorithm is applied to extract all the corner point from the image. After extracting all the corner points, the sliding window approach is used to find the most likely plate region.

The Harris corner detection algorithm is established along the point feature extraction of signal. It draws the window to move minute displacement in any direction. This algorithm determines the average changes of image intensity that result from shifting the window by a small amount in various directions. The change is given by:

$$
\begin{aligned}
& E_{x, y}=\sum_{u, v} W_{u, v}\left[\mathrm{I}_{\mathrm{x}+\mathrm{u}, \mathrm{y}+\mathrm{u}}-I_{u, v}\right]^{2} \\
& \sum_{u, v} W_{u, v}\left[\mathrm{xX}+\mathrm{y} \mathrm{Y}+\mathrm{O}\left(x^{2}, y^{2}\right]^{2}\right.
\end{aligned}
$$


Where $\mathrm{W}$ specifies the image window, it is unity within a specified rectangular region and zero elsewhere and I denotes the intensity of the image.

Where the first gradients are approximated by

$\mathrm{X}=\mathrm{I} \otimes(-1,0,1)=\partial \mathrm{I} / \partial \mathrm{x}$

$\mathrm{X}=\mathrm{I} \otimes(-1,0,1)=\partial \mathrm{I} / \partial \mathrm{y}$

Hence, for small shifts E can be written

$\mathrm{E}(\mathrm{x}, \mathrm{y})=\mathrm{A} x^{2}+2 C x y+W+\mathrm{B} y^{2}$

Where

$\mathrm{A}=\mathrm{X}^{2} \otimes \mathrm{W}$

$\mathrm{B}=\mathrm{Y}^{2} \otimes \mathrm{W}$

$\mathrm{C}=(\mathrm{XY}) \otimes \mathrm{W}$

By using above equations the symmetric matrix can be defined by

$$
\mathrm{M}=\left[\begin{array}{ll}
A & C \\
C & B
\end{array}\right]
$$

Then, The corner point can be extracted as the maximum of the local area

$$
\mathrm{R}=\operatorname{Det}(\mathrm{M})-\mathrm{kTr}^{2}(\mathrm{M})
$$

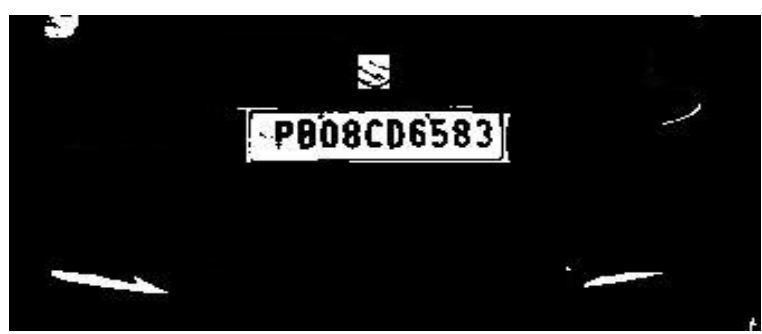

Fig 3: Multiple Probable Region

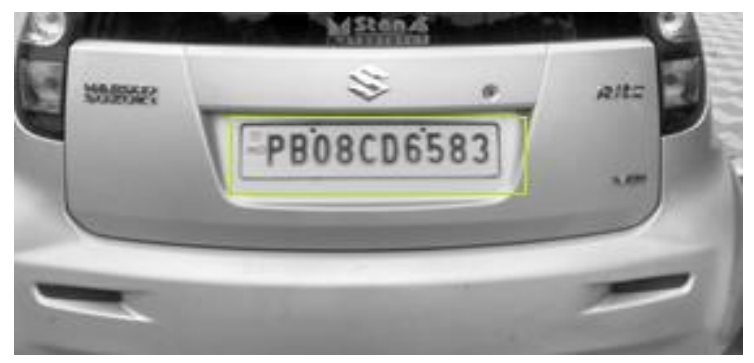

Fig 4: Localized License plate

\subsection{Tilt Correction}

In order to facilitate character segmentation and recognition in a vehicle license plate recognition system, the tilted license plate in the located image should be corrected. In this paper, LSFPO for fitted the license plate into a straight line. In this process first find the line slope and after that estimate the rotation angle between the principle axis $\mathrm{X}$ and the horizontal axis of the tilt license plate. Then the whole image is rotated by the rotation angle for tilt correction.

Fitting the straight line based on LFPO: The least square method is a generally used fitting method. Fitting needs a parametric model that relates the response data to the predictor data with one or more coefficients. To get the coefficient estimates, the least square method minimizes the summed square of residuals [11].
Fitting the straight line based on LSFVO: Given a set of data points. It is needed to search best fitting line from a given set of data points. In principle deviation between data and fitting line should be minimized [11].

After the successful fitting operation, calculate the rotation angle and the whole image is rotated by the rotation angle for tilt correction.

\subsection{Character Segmentation}

After the tilt correction the next step is character segmentation. It is a very important step because all the further steps depend on character segmentation. But, before segmentation, there is a need to enhance the image because of the contrast differences, which can occur while taking the image from the camera. Also, some unwanted elements can be present on the plate, which can decrease the performance of the system and affect the segmentation process. For enhancement the image the histogram equalization is used and median filter is used to remove the noise.

In character segmentation, in this paper the plate region is divided into blocks and characterize each and every data set as a single character. After the enhancement of the image, the adaptive thresholding is applied to make the license plate large. After the thresholding the connected component analysis (CCA) is applied to find the connected component from the license plate. These components may be character or non-character. To avoid these non-characters, which wrongly classified, aspect ratio is used. The aspect ratio is set based upon the observation for different types of results obtained from different images.

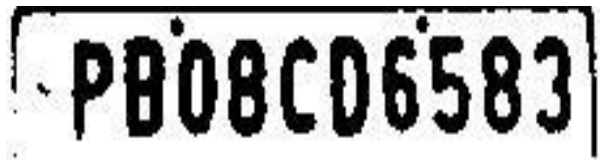

Fig 5: Extracted License plate

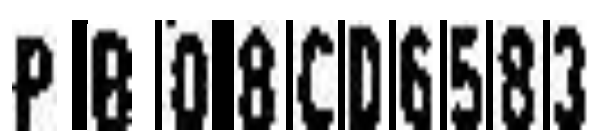

Fig 6: Segmented Characters

\subsection{Feature Extraction}

After character segmentation, each character is saved as a separate image file. Before character segmentation, the feature of the image should be extracted. The feature extraction process gives us the most selective information of an image and this information can be presented as a feature vector. This feature vector contains the local and global feature of the character, and these features should be encoded for this purpose Average Absolute Deviation algorithm is used. This algorithm is defined as:

$V=\frac{1}{N}\left(\sum_{N}|f(x, y)-M|\right)$

Where $\mathrm{N}$ is the number of pixels in the image, $\mathrm{m}$ is the mean of the image and $\mathrm{f}(\mathrm{x}, \mathrm{y})$ is the value at point $(\mathrm{x}, \mathrm{y})$. In our work, the character image is divided into sub-images and extracts the feature from each sub image by applying Average Absolute Deviation Algorithm. After applying this algorithm a feature vector is obtained for each character and then the entire feature vector is applied to the ANN as an input. 


\subsection{Character Recognition}

In this work, an artificial neural network is used for character recognition. In this paper a multi-layered perceptron (MLP) is used for classification of characters. Multi-layered perceptron is a single layered neural network which is organized in a cascading manner. It consists of an input layer, hidden layers, and an output layer.

For training, the following data is used: the input vector (feature vector), 36 classes (labels are 0-9 for digits and 0-25 for characters), and the output layer consists of 36 neurons and these are equal to the number of possible outputs (10 digits and 26 characters). Sigmoid function is used in the activation of neurons. For training this MLP, the backpropagation algorithm is used. The mean square error (MSE) is used to measure the training performance of the neural network. The value of the mean square error is used to verify how well the network output fits the desired output. MSE is used as stop criteria for training on a network. Most often the training set to terminate when the MSE drops to some threshold.

\section{RESULT ANALYSIS}

In order to evaluate the performance of the proposed system, 90 vehicle images were employed. Sigmoid function is used in the activation of neurons. The back propagation learning algorithm is used for training the artificial neural network. A maximum of 3000 iterations are performed for each input set. The mean square error is used as stop criteria for the training of the neural network. When the system is reached to the minimum error rate which is defined by the user, then the iteration is stopped. For our system the defined minimum error is 0.001 .

The success rate of the plate detection, character segmentation and character recognition of the proposed system are shown in Table 1. To evaluate the efficiency and robustness of proposed method, a database of 90 vehicle images in various illumination conditions have had been used. The Database contains the images captured at our college campus and some of the images from an online database to do evaluation on a larger scale. 85 license plates in 90 vehicle images were recognized correctly in our system, so the overall performance of the our system is $94.4 \%$.

Table 1: Result analysis

\begin{tabular}{|c|c|c|c|}
\hline Stage & $\begin{array}{c}\text { Number of } \\
\text { samples }\end{array}$ & $\begin{array}{c}\text { Number of } \\
\text { correct } \\
\text { results }\end{array}$ & $\begin{array}{c}\text { Success } \\
\text { Rate(\%) }\end{array}$ \\
\hline Detection & 90 & 88 & 97.78 \\
\hline Segmentation & 88 & 87 & 98.86 \\
\hline Recognition & $\begin{array}{c}880 \\
\text { characters }\end{array}$ & $\begin{array}{c}856 \\
\text { characters }\end{array}$ & 97.27 \\
\hline
\end{tabular}

\section{CONCLUSION}

In this paper proposed a system for vehicle license plate recognition based on Harris corner detection algorithm and artificial neural network. Our proposed system is able to correctly recognize the number plate of a vehicle image that is captured throughout the several timings and with diverse lighting conditions. This system is independent of the orientation of the plate. The proposed system is implemented in JAVA language and its performance is tested on a dataset of 90 image samples to recognize the number plates. Test results display that this method gives a good plate recognition rate.

\section{ACKNOWLEDGMENT}

I would like to thank to my Supervisor Mr. Deepak Kumar Gupta and my Co-Guide Mr. Raj Mohan Singh for continuous support and guidance. I also wish to thank whole heartedly all the faculty members of the Department of Computer Science and Engineering for the invaluable knowledge they have imparted on me and for teaching the principles in most exciting and enjoyable way.

\section{REFERENCES}

[1] License Plate Recognition, "How LPR works", http://www.licenseplatesrecognition.com/index.html;201 0

[2] Deb, K., Khan, I., Saha, A., \& Jo, K. H. 2012 An Efficient Method of Vehicle License Plate Recognition Based on iding Concentric Windows and Artificial Neural Network. Procedia Technology, 4, 812-819.

[3] Parasuraman, Kumar, and P. Vasantha Kumar 2010. An efficient method for indian vehicle license plate extraction and character segmentation. In IEEE International Conference on Computational Intelligence and Computing Research, pp. 1475-1477.

[4] Yu, Shouyuan, Baopu Li, Qi Zhang, Changchun Liu, and Max Q-H. Meng 2015. A novel license plate location method based on wavelet transform and EMD analysis. Pattern Recognition 48, no. 1 114-125.

[5] Neto, Edson Cavalcanti, Samuel Luz Gomes, Pedro Pedrosa Rebouças Filho, and Victor Hugo C. de Albuquerque 2015. Brazilian vehicle identification using a new embedded plate recognition system. Measurement 70 36-46.

[6] Nguwi, Y. Y., and W. J. Lim 2015. Number plate recognition in noisy image. In2015 8th International Congress on Image and Signal Processing (CISP), pp. 476-480. IEEE.

[7] Qiao, S., Zhu, Y., Li, X., Liu, T., \& Zhang, B. 2010. Research of improving the accuracy of license plate character segmentation. In 2010 Fifth International Conference on Frontier of Computer Science and Technology (pp. 489-493). IEEE.

[8] Jain, P., Chopra,N., Gupta, V. 2014. Automatic License Plate Recognition using OpenCV. International Journal of Computer Applications Technology and Research(pp. 756-761)

[9] Azad, Reza, Babak Azad, and Hamid Reza Shayegh 2014. Real-Time and Efficient Method for Accuracy Enhancement of Edge Based License Plate Recognition System. arXiv preprint arXiv:1407.6498.

[10] Al-Ghaili, Abbas M., Syamsiah Mashohor, Abdul Rahman Ramli, and Alyani Ismail 2013. Vertical-edgebased car-license-plate detection method. IEEE transactions on vehicular technology 62, no. 1 26-38.

[11] Deb, K., Khan, M. I., Alam, M. R., \& Jo, K. H. 2011. Optical Recognition of Vehicle license plates. In Strategic Technology (IFOST), 2011 6th International Forum on (Vol. 2, pp. 743-748). IEEE. 